\title{
Desenvolvimento e Validação de Escalas Brasileiras de Percepção e Internalização de Normas Corporais
}

\author{
Development and Validation of Brazilian Scales for Measuring \\ Perception and Internalization of Body Norms
}

\author{
Elizabeth Hirata ${ }^{*}$, , Amalia Raquel Pérez-Nebra ${ }^{\text {a, b }}$, \& Ronaldo Pilati ${ }^{\mathrm{a}}$ \\ ${ }^{a}$ Universidade de Brasília, Brasília, Brasil \& ${ }^{b}$ Centro Universitário de Brasília, Brasília, Brasil
}

\begin{abstract}
Resumo
O objetivo principal deste estudo foi adaptar, desenvolver e validar escalas brasileiras de normatização da aparência corporal para homens e mulheres. No Estudo 1 a escala SATAQ revelou uma estrutura bifatorial problemática. O Estudo 2 traz o desenvolvimento da EFIC/EMIC (Escala Feminina/Masculina de Normatização Corporal). Ambas apresentaram uma estrutura bifatorial, incluindo os fatores "internalização geral" (cargas fatoriais entre 0,85 e 0,37) e "intenção de comportamento" (cargas fatoriais entre 0,73 e 0,38). No Estudo 3 foram validadas juntas as escalas dos Estudos 1 e 2, acrescidos de análises correlacionais com dados demográficos. Observou-se relação entre ler, comentar e comprar produtos de beleza com a normatização da aparência corporal. A escala final agregou itens da SATAQ e EFIC/EMIC, mantendo os mesmos fatores da estrutura bifatorial.

Palavras-chave: Internalização, normas sociais, imagem corporal.

Abstract

The current research aimed at adapting, developing and validating Brazilian scales related to body image norms for both genders. In study 1, SATAQ revealed a problematic two-factor structure. Study 2 focused on the development of Male and Female Body Image Scales. Both scales revealed a two-factor structure, comprising the factors "general internalization" (factor loadings ranging from .85 to .37 ) and "behavioral intention" (factor loadings ranging from .73 to .38 ). In study 3 , combined scales from study 1 and 2 were validated. Besides that, correlational analyses with demographic data were performed. The analyses indicated a relation between reading, commenting and buying beauty products with body image norms. The final scale grouped SATAQ's and Male and Female Body Image Scales' items, keeping the same factors of the two-factor structure.

Keywords: Internalization, social norms, body image.
\end{abstract}

Na complexa dinâmica entre influência social e autoavaliação corporal, algumas variáveis têm mostrado uma importância crescente. A normatização e a internalização de padrões de corpo têm sido amplamente estudadas na literatura internacional. Pesquisas apontam a influência da internalização na relação entre padrões de corpo e avaliação corporal (Bessenoff \& Snow, 2006; Dittmar \& Howard, 2004; Durkin, Paxton, \& Sorbello, 2007).

Dentre as abordagens teóricas que estudam os padrões corporais, encontra-se a teoria sociocultural, levando em consideração o papel da cultura. A presente pesquisa se baseia majoritariamente nessa abordagem, buscando en-

\footnotetext{
*Endereço para correspondência: Departamento de Psicologia Social e do Trabalho, Universidade de Brasília, Campus Universitário Darcy Ribeiro, ICC Sul, Brasília, DF, Brasil 70910-900. E-mail: bethhir@gmail.com, pereznebra@gmail.com e rpilati@gmail.com. Elizabeth Hirata está agora em Jacobs University Bremen, Bremen, Alemanha.
}

riquecer a pesquisa relacionada à relação entre influência social normativa e imagem corporal individual no Brasil.

\section{Padrões de Corpo e Cultura}

A investigação científica sobre padrões de corpo e sua relevância social remete à antiguidade grega. Atualmente, a corrente da psicologia evolucionista busca por padrões universais, através das proporções de um corpo ideal (Etcoff, 1999). Alguns dos teóricos dessa abordagem estudam a atratividade corporal através de índices matemáticos de proporção corpórea, como o índice cintura-quadril (Tovée, Hancock, Mahmoodi, Singleton, \& Cornelissen, 2002). Perspectivas feministas também buscam entender a imagem corporal e os efeitos dos padrões corporais. A teoria da objetivação defende que a importância dos padrões de corpo está intimamente ligada com sua internalização. Na medida em que as mulheres os 
internalizam, elas os vivenciam como desejos internos, o que afeta a maneira como se percebem e lidam com tais normas (McKinley, 2002).

A teoria sociocultural defende a estreita ligação dos padrões de corpo com o meio cultural (Groesh, Levine, $\&$ Murnen, 2002). Entende-se que a cultura exerce um papel fundamental na formação dos padrões de corpo considerados ideais, bem como no desenvolvimento e oscilações da imagem corporal das pessoas (Jackson, 2002). Com base nessa teoria, busca-se avaliar quais padrões de corpo são considerados ideais em diversos países e seu impacto na satisfação corporal (ver metaanálise de Groesh et al., 2002).

Partindo-se da importância da cultura no entendimento das influências sociais na imagem corporal do indivíduo, salienta-se a relevância de estudos feitos em diversos contextos culturais. No contexto nacional, estudos apontam haver relação entre aspectos do self ${ }^{1}$, a aparência física e o corpo (Giavoni \& Tamayo, 2005; Tamayo, 1981). A aparência física desponta como uma característica de grande importância no país, o que pode ser ilustrado pelas taxas de cirurgias estéticas e pela venda de remédios para emagrecer, que estão entre os primeiros lugares do mundo, de acordo com a Sociedade Brasileira de Cirurgia Plástica (SBCP, 2004) e a Junta Internacional de Fiscalização de Entorpecentes (JIFE, 2007).

Padrões de corpo têm sido entendidos como normas sociais, que, de acordo com Cialdini e Trost (1998), são definidas como regras negociadas pelo grupo social e que dizem respeito à regulação de condutas esperadas pelos membros do grupo. Dois processos subjazem a relação individual com tais normas sociais. Um deles refere-se à percepção da norma, ou seja, a percepção do indivíduo sobre a existência e compartilhamento social de uma regra. O outro trata da internalização da norma. As teorias de internalização descrevem um continuum no qual valores sociais ou regras são adotados como algo pertencente ao self ou com o qual o indivíduo se identifica. Quanto mais internalizado um valor ou regra, mais ele é vivenciado como autônomo ou subjetivamente próximo do self (Ryan \& Connell, 1989).

Na pesquisa em imagem corporal, a normatização da magreza feminina tem sido amplamente estudada. Diversos estudos evidenciam que o padrão feminino ideal é a norma de magreza, com um corpo magro e definido (e.g. Dittmar \& Howard, 2004; Grogan, 2008; Hirata, 2009; Park, 2005). O ideal masculino é o de musculosidade, mostrando um homem com o abdômen definido, músculos salientes e corpo em forma de "V" (Grogan, 2008; Harvey \& Robinson, 2003; Labre, 2002).

\footnotetext{
${ }^{1}$ Entende-se aqui o complexo fenômeno do self a partir de três raízes principais que englobam diversos construtos relacionados: a consciência reflexiva, o self interpessoal e, por fim, a função executiva. Sugere-se Baumeister (1998), para um entendimento mais aprofundado do tema.
}

Thompson e Stice (2001) tratam do ideal de magreza, definindo a sua internalização como o "nível no qual um indivíduo adere cognitivamente aos ideais socialmente definidos de atratividade e adquire comportamentos adequados para se aproximar dos mesmos" (p. 181). Segundo Ford, Stevenson, Wienir e Wait (2002), conforme as pessoas internalizam os padrões de corpo, identificandose e guiando-se por eles, elas podem passar a perceber discrepâncias entre si mesmas e os padrões compartilhados, o que pode desencadear em pioras nas avaliações afetivas de si mesmo.

As normas, além de estarem relacionadas ao self, também podem ser desencadeadoras de comportamentos (e.g. Aarts, Dijksterhuis, \& Custers, 2003; Cialdini \& Goldstein, 2004). A ativação de normas corporais tem impacto em comportamentos relativos ao corpo, como por exemplo, o comportamento alimentar (Harrison, Taylor, \& Marsk, 2006; Strahan, Spencer, \& Zanna, 2007). Não foram encontrados estudos nacionais relacionando padrões corporais com comportamentos relacionados.

O estudo da normatização da aparência corporal apresenta relevância social e clínica, particularmente no caso de indivíduos com distúrbios relacionados à aparência, ou à imagem corporal. Considerando-se as implicações do fenômeno da norma social para o estudo dos processos relativos ao corpo, faz-se necessário desenvolver instrumentos de medida válidos para a pesquisa e a prática profissional em nosso contexto cultural.

Entretanto, o entendimento no contexto nacional oferece algumas dificuldades, como a adaptação das normas sociais locais e a mensuração do que é belo, aceito, desejado, e o que se faz para atingir estes ideais. O estudo de aspectos normativos da aparência corporal no país requer o uso de medidas confiáveis e adaptadas.

\section{Medidas de Normatização Corporal}

A literatura internacional oferece algumas medidas de internalização, das quais a mais conhecida e utilizada é o Questionário Sociocultural das Atitudes sobre a Aparência (Sociocultural Attitudes Towards Appearance Questionnaire [SATAQ] de Heinberg, Thompson, \& Stormer, 1995). O instrumento foi criado em consonância com a teoria sociocultural. Ele possui dois fatores nomeados Internalização, referente à aceitação dos padrões e Consciência, relativa ao reconhecimento do padrão, de uma ênfase social na aparência. Seus respectivos alfas de Cronbach foram de 0,93 e 0,81 no primeiro estudo de validação e de 0,88 e 0,71 no segundo.

Posteriormente foi criada a Sociocultural Attitudes Towards Appearance Questionnaire-3, (SATAQ-3; Thompson, Van den Berg, Roehrig, Guarda, \& Heinberg, 2004). Dentre seus fatores, a escala apresentou dois de internalização: o Internalização Geral e Internalização Atleta, com os alfas de 0,96 e 0,95 na primeira amostra e de 0,92 e 0,89 na segunda. 
A escala SATAQ-3 tem algumas diferenças em relação à SATAQ. Em relação à SATAQ original, a versão 3 torna ainda mais explícita nos seus itens a influência da mídia, por exemplo, enfatizando a comparação e a relação individual com modelos advindos da TV do cinema e outras fontes midiáticas. Ela inclui, ainda, um foco no aspecto atlético das mulheres, examina outras influências da mídia, além da internalização, como as pressões e informações da mídia e avalia a diferença do construto internalização com outras escalas. Assim, a internalização e aspectos da mídia, como a pressão e informações, têm sido estudados pela literatura internacional com as escalas supracitadas.

A SATAQ e SATAQ-3 foram utilizadas em diversas pesquisas (e.g. Dittmar \& Howard, 2004; Durkin et al., 2007; Forbes et al., 2005). Entretanto, não foram encontradas traduções ou escalas que medissem o construto internalização em língua portuguesa. Nesse ponto, menciona-se a primeira justificativa do presente estudo; a de oferecer um instrumento de internalização adaptado ao contexto nacional. A proposta inicial foi adaptar a SATAQ para possibilitar comparações com a literatura internacional (Estudo 1).

A SATAQ e SATAQ-3 medem a internalização através de itens como "Eu comparo meu corpo com o corpo de modelos", "Eu freqüentemente leio revistas como Nova, Vogue, e Marie Claire e comparo a minha aparência com a das modelos" ou "Eu gostaria de parecer tão atlético(a) como as pessoas nas revistas". Nota-se que os itens citam explicitamente a fonte de divulgação e reforço desses padrões ao falar de "pessoas na televisão", "modelos" e "Vogue e Marie Claire", por exemplo.

Contudo, algumas pessoas podem ter internalizado os padrões preconizados pela mídia, sem acreditar se comparar ou se inspirar em modelos ou atores. O indivíduo assimila o conteúdo dos padrões, sem que a fonte, influências da mídia, esteja acessível em sua memória. Entende-se que uma norma cultural foi internalizada quando um padrão público ou noção coletiva se tornaram um ideal privado da pessoa- um padrão que a pessoa acredita ser originado de dentro do self (Bessenoff $\&$ Snow, 2006). Assim, é possível que quando o indivíduo internaliza o padrão de corpo, ele traga para si apenas seu conteúdo-magreza para as mulheres e musculosidade para homens- sem que o associe diretamente à fonte de origem.

Ademais, os aspectos comportamentais relativos à norma não tem recebido tanta atenção. Não foram encontradas medidas avaliando a intenção de comportamento em relação às normas de aparência corporal. $\mathrm{O}$ que se encontrou foi o Questionário Muldimensional das Relações Self-Corpo (Cash, 2000), que possui alguns fatores medindo aspectos comportamentais, a partir da sub-escala de orientação à aparência, saúde, doença e boa-forma. Seus itens avaliam comportamentos já existentes, de investimento comportamental no corpo.
Desse modo é útil desenvolver uma medida que afira não somente o quanto uma pessoa internaliza de maneira consciente que o padrão construído advém de uma fonte externa, mas também indague puramente sobre os conteúdos da norma. Seria interessante mensurar também em que medida as pessoas endossam o que se define como padrão e o quanto elas se comportam de modo coerente a ele. Assim, a medida estaria avaliando um ponto da normatização corporal ainda não contemplado na escala SATAQ, o aspecto da intenção comportamental.

Finalmente, a literatura de imagem corporal tem dado menos atenção ao corpo masculino do que o feminino (Labre, 2002). A internalização e a percepção da norma de corpo feminina têm sido continuamente estudadas (e.g. Dittmar \& Howard, 2004; Durkin \& Parkinston, 2002; Durkin et al., 2007; Tiggemann \& McGill, 2004). Tais aspectos começam a ser estudados de modo mais sistemático em relação aos homens, embora em menor escala (Dittmar, 2008). O estudo de aspectos normativos da imagem corporal masculina pode ser beneficiado com a presença de instrumentos de aderência do fenômeno.

Assim, este trabalho tem como objetivo validar uma escala de norma social de aparência corporal para homens e mulheres, além de relacionar a escala de normas sociais da aparência com dados demográficos. Para atingir o objetivo principal foram realizados os seguintes estudos:

1. Tradução e avaliação da validade do SATAQ no contexto brasileiro com os fatores originais de percepção e internalização de normas sociais com a aparência.

2. Desenvolvimento e validação de um instrumento que desdobra o conceito de internalização e de intenção comportamental, na versão feminina (EFIC) e masculina (EMIC).

3. Junção das escalas para obter uma escala brasileira híbrida que contenha os itens novos e antigos.

\section{Estudo 1}

Partindo-se da teoria sociocultural, que embasa as atuais escalas de internalização, salienta-se a importância do estudo do fenômeno, em culturas distintas (Grogan, 2008). Até o presente momento, não foram encontradas escalas validadas no Brasil que avaliem a influência normativa dos padrões no indivíduo. O entendimento desse fenômeno no país pode ser favorecido com a disponibilização de medidas válidas que facilitem a pesquisa na área.

Visando possibilitar mais ferramentas à pesquisa em imagem corporal, com base no entendimento normativo do corpo, o presente estudo buscou adaptar e validar a escala SATAQ, que indaga sobre o reconhecimento e a aceitação dos padrões corporais. Objetivou-se avaliar a validade dessa escala estadunidense no contexto brasileiro. Essa escala foi utilizada em estudos anteriores e 
Hirata, E., Pérez-Nebra, A. R. \& Pilati, R. (2012). Desenvolvimento e Validação de Escalas Brasileiras de Percepção e Internalização de Normas Corporais.

uma versão traduzida seria útil ao permitir comparações com estudos internacionais que a utilizaram.

\section{Método}

Participantes. Participaram 960 usuários de Internet, das 5 regiões do Brasil. Desses, $72 \%$ eram mulheres. Eles foram contatados a partir de e-mails de divulgação de pesquisa, a partir de listas de contato. A média de idade foi de 33,1 anos $(D P=11,5), 42,2 \%$ de casados e $34,1 \%$ de solteiros. $42,8 \%$ recebem menos de 2 mil reais mensais e $45,6 \%$ se declararam brancos.

Instrumento. $\mathrm{O}$ instrumento apresentado aos participantes foi o SATAQ original. O instrumento original proposto por Heinberg et al. (1995) é composto por 14 itens. Desses, 8 itens refere-se ao fator Internalização, com um item reverso. O fator Percepção da Norma Social contém 6 itens, com 3 reversos. Foi realizada a tradução e a revisão dos itens por 6 especialistas em psicologia, fluentes em português e inglês. Os itens estão associados a uma escala Likert de 5 pontos e as cargas fatoriais de validação relatadas pelos autores variam de 0,71 a 0,88 .

Procedimentos de Coleta e Análise de Dados. Os participantes responderam a um questionário online com o método de bola de neve (Snijders, 1992). Os respondentes recebiam um e-mail, entravam em um sitio eletrônico, e a primeira página visitada descrevia o Termo de Consentimento Livre e Esclarecido. Uma vez aceitas as condições de pesquisa, eles acessavam a página seguinte e respondiam ao questionário.

Foram realizadas análises descritivas para verificar dados omissos sistemáticos, assimetria e curtose. Não ocorreu sistematicidade de casos omissos. Alguns itens apresentaram problemas de assimetria e outros de curtose, e apenas o Item 6 apresentou problemas em ambos os indicadores. Optou-se por mantê-los, sem realizar correções. A manutenção destes itens na análise deu-se porque não se obteve melhor simetria, tendo a transformação apenas alterado a distribuição de leptocurtica para platocurtica e de assimetria positivia para negativa. As análises realizadas incluíram toda a amostra, pois esta escala é considerada, pelos autores, adequada a mulheres e homens. Análises apontaram a fatorabilidade da escala, que apresentou um determinante de 0,005 e KMO de 0,85. A estrutura fatorial foi analisada por Fatoração dos Eixos Principais (PAF) com rotação obliqua oblimin.

\section{Resultados}

A análise da extração de fatores indicou que uma estrutura unifatorial explicava suficientemente os dados (auto-valor superior a 2 e variância explicada de 32,1\%). A análise paralela de Horn indicou a extração de cinco fatores. Entretanto, como não havia sentido teórico em tal estrutura, buscou-se testar a estrutura empírica original da escala. Então, foi realizada uma análise fatorial com extração de dois fatores. Os resultados indicam inadequação desta estrutura fatorial, pois o fator Internalização replica o original, mas o fator Percepção manteve apenas quatro dos seis itens originais. A análise de consistência interna indica que apenas o fator Internalização é adequado (alfa $=0,88)$, pois Percepção da norma alcança índice de consistência insuficiente (alfa $=0,63$ ).

\section{Discussão}

O presente estudo aponta limitações da escala para uso no Brasil. Pretendia-se manter a escala original, conforme relatado por Heinberg et al. (1995), para facilitar comparações com estudos internacionais. Entretanto, ela se mostrou inadequada pois a estrutura bifatorial não foi corroborada. Além da estrutura fatorial não ter sido corroborada também foi observado índice de consistência interna insuficiente (Pasquali, 2003). Estes resultados indicam que é necessário empreender um aprimoramento da medida para o contexto cultural brasileiro. Tal resultado não surpreende na medida em que se entende a cultura como um elemento central nas oscilações da imagem corporal. Oscilações entre preferências corporais são encontradas ao longo do tempo e em diferentes contextos culturais (Grogan, 2008; Jackson, 2002). Dessa forma, não se surpreende que haja também diferenças em medidas avaliando efeitos da normatização corporal.

A análise da escala indica que dimensões relevantes de avaliação do construto não são contempladas SATAQ. Observa-se como necessário superar limitações da escala original, como, por exemplo, a ausência de um fator relacionado a descrição de intenção comportamental da norma. Conforme salientado anteriormente, a exposição à norma de corpo é um fator que pode alterar determinados comportamentos (Harrison et al., 2006; Strahan et al., 2007). Dessa forma, incluir o aspecto da intenção de comportamento, propiciaria um ganho no entendimento do fenômeno da normatização corporal e o indivíduo. Ademais, a inserção de mais uma dimensão desta natureza pode facilitar uma possível comparação com os estudos internacionais sobre normas sociais com a aparência, o que diminuiria o problema da impossibilidade de uso de uma versão em português da escala.

Resumidamente, no Estudo 1, não foi corroborada a estrutura empírica da SATAQ numa amostra de brasileiros, o que apontou a pertinência em se desenvolver uma nova medida. Essa escala poderia aferir não somente internalização, mas também a intenção comportamental. Com vistas a desenvolver a nova medida do fenômeno com a implementação da nova dimensão procedeu-se um estudo adicional. Esse estudo teve como objetivo responder às limitações indicadas por meio da criação de novos itens, principalmente para o desdobramento do fator relativo à internalização das normas sociais.

\section{Estudo 2}

Dada a limitação da escala SATAQ para o Brasil, propôs-se uma medida que pudesse ser utilizada tanto em homens quanto em mulheres e em diversos tipos de amos- 
tra, inclusive foram do contexto clínico, para fins de pesquisa. A SATAQ original foi formulada pensando nos padrões de magreza em pacientes com transtorno alimentar. A SATAQ-3 foi validada inicialmente com duas amostras femininas. Desse modo, embora tenham indicadores psicométricos muito bons, os instrumentos não foram elaborados em coerência com o objetivo supracitado.

Desse modo, o objetivo do Estudo 2 foi o desenvolvimento e validação de duas escalas, uma masculina e outra feminina, para avaliação de internalização da norma de corpo, assim como de intenção comportamental em relação à norma.

\section{Método}

Participantes. Participaram do estudo 451 estudantes universitários, a maioria de instituição pública $(64,7 \%)$, alunos de diversos cursos, com 55,7\% da amostra composta por mulheres. Trata-se de uma amostra de conveniência. A média de idade foi de 24 anos $(D P=6,7)$.

Instrumento. Foram adaptados itens da SATAQ e SATAQ-3 e propostos novos itens para as escalas, elaboradas em duas versões, uma feminina e outra masculina. Estas foram, respectivamente, nomeadas como EFIC (Escala Feminina de Internalização Corporal) e EMIC (Escala Masculina de Internalização Corporal). Os itens das escalas estão apresentados nas Tabelas 1 e 2 . Não foi utilizada a técnica de tradução-retradução, pois o estudo não buscou traduzir o instrumento, mas adaptar e propor um novo instrumento. Assim, as traduções foram feitas por especialistas bilíngües da área de psicologia. Seguiram-se diversas validações semânticas que delinearam a forma final da escala. A validação semântica seguiu as instruções de Pasquali (2003). Vinte participantes responderam a uma versão inicial do instrumento traduzido. Essas pessoas relataram o entendimento de cada item e sugeriram algumas mudanças para sua melhoria. Em seguida, foi realizada a validação por especialistas, com 5 psicólogos que dividiram os itens em função dos construtos, tais como o percebiam. Dessa maneira, chegou-se à versão final do instrumento.

A escala buscou medir dois aspectos relevantes da normatização de padrões de corpo, a internalização dos padrões e a descrição de comportamentos de conformidade aos mesmos. Desse modo, hipotetizou-se que essas seriam as duas dimensões principais da escala, o que guiou a escolha dos itens.

Para a proposição da definição operacional, entendese que a internalização se refere à aceitação dos padrões (Heinberg et al., 1995). A norma cultural é internalizada na medida em que um padrão público ou noção coletiva são incorporados a um ideal privado, algo que ela julga ser originado de dentro do self (Bessenoff \& Snow, 2006). Partiu-se do conhecimento que a norma para as mulheres, grosso modo, é a magreza e para homens, a musculosidade. Assim, a internalização foi definida operacionalmente como o endosso ou a aceitação de padrões de corpo ideal. Entendeu-se aceitação como apreciação e desejo de possuir um corpo semelhante aos padrões de corpo, definidos em torno da magreza, massa e definição muscular e rejeição à gordura, variando em função do sexo. $\mathrm{O}$ aspecto comportamental buscou medir esforço ou intenção comportamental para se adequar aos padrões, entendido como uma tentativa de conformidade normativa. Ele foi operacionalizado em função de intenções de comportamentos ou desejos de aproximação dos padrões de corpo ideal, como dietas, exercícios, cirurgias plásticas e outros tratamentos estéticos.

A versão masculina do instrumento ficou semelhante à feminina, sofrendo algumas mudanças de adaptação ao gênero. Como o padrão masculino é o de musculosidade, itens que falavam de "mulheres magras", na escala masculina, foram substituídos por "homens musculosos".

Procedimento de Coleta e Análise de Dados. Os dados foram coletados em horários distintos. Após a autorização dos professores, os aplicadores solicitavam a participação dos alunos em sala de aula. Houve também coleta individual de dados nos corredores das instituições. Em ambos os casos, os alunos recebiam um Termo de Consentimento Livre e Esclarecido. Os dados foram submetidos a uma análise de componentes principais seguida da análise fatorial exploratória pelo método de fatoração dos eixos principais (PAF). As análises foram feitas separadas por sexo, por se tratar de duas medidas distintas. Conforme sugerido por Tabachnick e Fidell (2007), manteve-se os itens com cargas fatoriais mínimas de 0,32 .

\section{Resultados}

A amostra feminina (EFIC) apresentou um bom índice de fatorabilidade da matriz, com o KMO de 0,89 e o determinante foi próximo a zero. Na masculina o KMO de 0,85 e o determinante foi também próximo a zero. A análise do gráfico de sedimentação, apontou entre 2 a 4 fatores. A análise paralela (Enzmann, 1997) apontou 2 fatores. A estrutura bifatorial explicou $49 \%$ da variância total da matriz, enquanto que um acréscimo de 2 fatores aumentaria essa variância em apenas $12,7 \%$. Desse modo, optou-se por testar uma estrutura bifatorial, o que confirmaria a estrutura hipotetizada de duas dimensões principais.

$\mathrm{Na}$ escala masculina, EMIC, o gráfico de sedimentação apontou entre 2 e 4 fatores. Assim como na EFIC, houve 4 autovalores acima de 1, indicando a possibilidade de se extrair até 4 componentes. A análise paralela da EMIC apontou 3 fatores. A análise da estrutura de 3 fatores revelou que um terceiro fator com apenas 3 itens, tendo 1 deles saturado em dois fatores, com cargas fatoriais próximas. Testou-se uma estrutura bifatorial, que além de condizer com a estrutura esperada, em torno de dois eixos principais, apresentou melhores indicadores de confiabilidade. Somados, os dois fatores explicam quase $50 \%$ do total da variância. $\mathrm{O}$ acréscimo de 1 fator aumentaria $7,7 \%$ essa variância. Desse modo, optou-se pela escolha da estrutura bifatorial da escala. 
$\mathrm{Na}$ EFIC, o primeiro fator possuiu 10 itens com cargas fatoriais variando entre 0,48 e 0,83 (ver Tabela 1 ). Seu alfa de Cronbach foi de 0,81 e a correlação item-total média, de 0,60 $(D P=0,11)$. Os itens "Mulheres que aparecem na TV e no cinema possuem o tipo de corpo que vejo como meu objetivo" e "Eu gostaria que meu corpo parecesse com o de mulheres famosas, como atrizes ou modelos" ilustram o conteúdo do fator Internalização. Esse fator agregou, ainda, itens de internalização que não incluíam menções à mídia, como o "Para mim, o ideal seria ter um corpo magro e definido".
O segundo fator da EFIC obteve 6 itens com cargas fatoriais variando de 0,45 a 0,73 . Seu alfa foi de 0,87 e sua correlação item-total média, de $0,54(D P=0,07)$. Os itens "Eu faria exercícios para ter menos gordura corporal" e "Eu faria exercícios para ficar mais definida" obtiveram as maiores cargas fatoriais, de 0,73 e 0,62 , respectivamente. Os outros itens desse fator tratavam de aspectos comportamentais relativos aos padrões, como o item de maior carga fatorial, "Eu faria dietas para ter menos gordura corporal". Assim, o fator foi intitulado Intenção de Comportamento.

Tabela 1

Cargas Fatoriais da Escala Feminina de Normatização de Padrões de Corpo- EFIC

\begin{tabular}{rrr}
\hline Item & Item & Cargas \\
& Fatoriais & Comunalidades \\
\hline
\end{tabular}

Fator 1: "Internalização" $(\alpha=0,81)$

2 Mulheres que aparecem na TV e no cinema possuem o tipo de corpo que vejo como meu objetivo

18 Eu gostaria que meu corpo parecesse com o de mulheres famosas, como atrizes ou modelos

$4 \quad$ Eu gostaria de parecer tão atlética quanto as pessoas em revistas e na TV

12 Os programas de TV e cinema mostram mulheres magras que me fazem querer ser mais magra

14 Ver imagens de mulheres magras me faz querer estar magra

16 Para mim, o ideal seria ter um corpo magro e definido

15 Eu faria tratamentos estéticos para melhorar a aparência do meu corpo

8 Eu gostaria de parecer tão atlética quanto as atletas profissionais

3 Eu compraria produtos para melhorar minha aparência física

9 Eu faria cirurgias para melhorar a aparência do meu corpo

$\begin{array}{ll}0,83 & 0,65 \\ & \\ 0,83 & 0,60 \\ 0,82 & 0,60 \\ & \\ 0,66 & 0,53 \\ 0,63 & 0,53 \\ 0,51 & 0,39 \\ 0,49 & 0,42 \\ 0,49 & 0,23 \\ 0,49 & 0,26 \\ 0,48 & 0,22\end{array}$

Fator 2: "Intenção de comportamento" $(\alpha=0,87)$

11 Eu faria exercícios para ter menos gordura corporal

$5 \quad$ Eu faria exercícios para ficar mais definida

13 Atualmente faço exercícios para melhorar minha aparência física

10 Eu estou sempre tentando melhorar minha aparência corporal

1 Eu faria dietas para ter menos gordura corporal

6 Atualmente faço dietas para melhorar minha aparência física

$\begin{array}{ll}0,73 & 0,55 \\ 0,62 & 0,35 \\ 0,57 & 0,28 \\ 0,52 & 0,49 \\ 0,49 & 0,49 \\ 0,45 & 0,32\end{array}$

O primeiro fator da EMIC foi composto por 8 itens, com cargas fatoriais entre 0,37 e 0,85 . Seu alfa foi de 0,88 e sua correlação item-total média de 0,65 $(D P=0,11)$. Os itens "Eu gostaria que meu corpo parecesse com o de homens famosos, como atores ou modelos" e "Os programas de TV e cinema mostram homens fortes que me fazem querer ser mais musculoso" obtiveram as maiores cargas fatoriais, de 0,85 e 0,77 , respectivamente. O conteúdo dos itens nesse fator se agrupou em função dos padrões de corpo da mídia e de alguns comportamentos de conformidade, em especial cirurgia, tratamentos estéticos e compra de produtos. O agrupamento desses conteúdos referem-se à Internalização da Norma Social.
O segundo fator da EMIC, com 9 itens, obteve cargas fatoriais variando entre 0,38 e 0,69 . Seu alfa foi de 0,81 e sua correlação item-total média, de $0,52(D P=0,09)$. As cargas fatoriais mais altas foram dos itens "Atualmente faço exercícios para melhorar minha aparência física" e "Eu estou sempre tentando melhorar minha aparência corporal", de 0,69 e 0,68, respectivamente. Esse fator incluiu também itens sobre padrão de corpo, relativos a musculosidade e atletismo. Esse resultado aponta que a intenção comportamental com dietas e exercícios está relacionada à busca de musculosidade e de um corpo definido. Contudo, a nomeação do fator como Intenção de Comportamento foi feita em função da maioria das questões e daquelas com as maiores cargas fatoriais (Tabela 2). 
Tabela 2

Cargas Fatoriais da Escala Masculina de Normatização de Corpo- EMIC

\begin{tabular}{lcc}
\hline Item & Item & Cargas \\
& Fatoriais \\
\hline
\end{tabular}

Fator 1: "Internalização" $(\alpha=0,88)$

18 Eu gostaria que meu corpo parecesse com o de homens famosos, como atores ou modelos $\quad 0,85$

$\begin{array}{ll}0,85 & 0,65 \\ 0,77 & 0,71 \\ 0,76 & 0,65 \\ & \\ 0,67 & 0,55 \\ 0,66 & 0,40 \\ 0,62 & 0,52 \\ 0,60 & 0,31 \\ 0,37 & 0,34\end{array}$

Fator 2: "Intenção de comportamento" $(\alpha=0,81)$

13 Atualmente faço exercícios para melhorar minha aparência física

10 Eu estou sempre tentando melhorar minha aparência corporal

11 Eu faria exercícios para ter menos gordura corporal

$7 \quad$ Acho importante ter um corpo definido

$5 \quad$ Eu faria exercícios para ficar mais musculoso

16 Para mim, o ideal seria ter um corpo musculoso

6 Atualmente faço dietas para melhorar minha aparência física

1 Eu faria dietas para ter menos gordura corporal

8 Eu gostaria de parecer tão atlético quanto os atletas profissionais

$\begin{array}{ll}0,69 & 0,41 \\ 0,68 & 0,49 \\ 0,68 & 0,40 \\ 0,65 & 0,46 \\ 0,53 & 0,39 \\ 0,46 & 0,46 \\ 0,44 & 0,22 \\ 0,39 & 0,16 \\ 0,38 & 0,32\end{array}$

\section{Discussão}

O objetivo deste estudo foi desenvolver e validar a versão feminina e masculina de uma escala de ideais de corpo. Os instrumentos EFIC e EMIC se mostraram válidos. A estrutura bifatorial foi comum às duas escalas. Ambas foram entendidas em função da internalização e da intenção comportamental relativa a exercícios e dietas, com alguma diferença entre elas na composição dos itens.

Em ambas as escalas e em ambos os fatores, o fator foi nomeado em função dos itens de maior carga fatorial. A divisão exata dos fatores em função da esfera comportamental e da internalização não ocorreu exatamente como esperado. Alguns itens de Internalização saturaram no fator relativo a comportamento e vice-versa. Essa levou a realização de um terceiro estudo que combinou a escala original do SATAQ a estes dois fatores relativos à Internalização Geral e de Intenção de Comportamento. O objetivo foi obter uma escala de norma social que seja comparável internacionalmente e completa, incluindo itens relativos a percepção, a internalização, e intenção de comportamento em relação à norma social.

Itens relativos à norma cujo conteúdo versava sobre a mídia agruparam-se com itens que não explicitavam a fonte da norma. Itens como "Mulheres que aparecem na TV e no cinema possuem o tipo de corpo que vejo como meu objetivo" e "Para mim, o ideal seria ter um corpo magro e definido" apareceram no mesmo fator, Estudos futuros, com amostras distintas poderiam averiguar se tais itens tenderiam a se agrupar em amostras distintas.

Observou-se uma diferença entre os fatores de intenção comportamental da escala feminina e masculina. $\mathrm{Na}$ versão masculina, o item mais representativo foi o "Atualmente faço exercícios para melhorar minha aparência física". Na feminina figurou o "Eu faria exercícios para ter menos gordura corporal." Nota-se a ênfase da questão da gordura na intenção comportamental da mulher. Tal resultado pode ser entendido à luz da importância da magreza na imagem corporal feminina, que tem sido enfatizada (Forbes et al., 2005; Groesh et al., 2002; Park, 2005; Strahan et al., 2007). Elas estão menos satisfeitas em relação à sua gordura do que os homens (Hirata \& Pilati, 2010).

Tendo em vista que o padrão feminino ideal é a norma de magreza (Dittmar \& Howard, 2004; Grogan, 2008; Hirata, 2009; Park, 2005), observa-se o agrupamento dos conteúdos em relação à internalização da norma. Entretanto, na escala masculina, o item mais representativo do 
Hirata, E., Pérez-Nebra, A. R. \& Pilati, R. (2012). Desenvolvimento e Validação de Escalas Brasileiras de Percepção e Internalização de Normas Corporais.

fator intenção de comportamento não abordou a musculosidade, mas a aparência física de modo geral. Segundo Strahan et al. (2007), enquanto o padrão de corpo feminino é rigorosamente o padrão de magreza, o homem para ser considerado atraente pode ter uma gama maior de tipos corporais.

A análise dos itens com maior carga fatorial das escalas feminina e masculina aponta à distinção entre a internalização da norma e os comportamentos relacionados se diferem. Esse achado aponta à distinção entre internalização e a intenção comportamental relativos à normatização corporal. Embora não exclusivamente, a intenção comportamental pode ser entendida como a busca de conformidade aos padrões de corpo, ao se falar de exercícios, dietas, consumo de produtos estéticos, de cirurgias plásticas, remédios para emagrecer, dentre outros. Segundo Deutsch e Gerard (1955), uma vez que uma norma tenha sido internalizada ela se torna parte integrante do auto-conceito da pessoa, e comportamentos normativos futuros representarão conformidade às próprias expectativas do self, levando a sentimentos de auto-estima ou auto-aprovação. E comportamentos de conformidade, são aqueles que buscam aproximar os indivíduos da norma. Desse modo, comportamentos como dietas e exercícios nesse intuito, mostram-se uma importante faceta da relação normativa entre influência social e imagem corporal. Ao falar da influência social percebida, Cafri, Yamamiya, Brannick e Thompson (2005) mencionam a consciência de um padrão de corpo socialmente partilhado e a internalização dessa norma. A última pode chegar ao ponto de afetar o comportamento pessoal.

Uma limitação do estudo foi relativa a amostra, restrita apenas a estudantes universitários. Além disso, os itens não se agruparam todos de acordo com o esperado. Alguns itens que não incluíam a menção a fontes de mídia se agruparam no fator de intenção comportamental. Dessa forma, para reavaliar o construto, optou-se por realizar um terceiro estudo para aprimorar a medida.

\section{Estudo 3}

O presente estudo agregou os itens originais da SATAQ e os novos itens relativos à internalização e intenção e comportamento da EMIC e da EFIC, de forma a se obter uma medida mais fidedigna da definição do que é uma norma social de aparência.

\section{Método}

Participantes. Responderam ao instrumento 960 usuários de Internet. Destes, $72 \%$ eram mulheres. A média de idade foi de 33,1 anos $(D P=11,5)$. Os participantes respondiam em primeiro lugar a escala SATAQ original e na sequência os itens da EFIC/EMIC.

Instrumento. $\mathrm{O}$ instrumento foi composto pelo SATAQ original, incluindo os itens com cargas fatoriais aceitáveis no Estudo 2. Também foram incluídos dados demográficos. A diferença no instrumento foi na adaptação da escala masculina onde os itens que descreviam musculosidade foram substituídos por atletismo.

Procedimento de Coleta e Análise de Dados. O procedimento de coleta e análise de dados foram os mesmos do Estudo 1, à exceção de análises separadas por sexo feitas neste estudo. Esse procedimento foi realizado, uma vez que os itens incluídos, por serem diferentes entre os sexos, não permitem que a análise seja feita em conjunto. O critério de carga fatorial utilizado neste estudo foi um pouco mais severo que no estudo anterior. Optou-se pelo critério de 0,40 , com apenas uma exceção com carga 0,39 por ser um item considerado muito típico. Para a análise de relação entre as variáveis demográficas e os fatores de norma sociais com aparência foram realizadas análises de correlação e ANOVA de uma via.

\section{Resultados}

Escala Masculina. As análises de fatoração apontam para a possibilidade de agregação dos itens, já que o KMO foi de 0,94 , e o determinante foi muito próximo de zero. A análise do auto-valor apresenta dois fatores superiores a 2, mas três fatores com porcentagem de variância explicada acima de $3 \%$.

Conforme o esperado procedeu-se com a análise de dois e três fatores. A extração de três fatores apresentou-se inviável, já que o último fator apresentava três itens, com baixas cargas fatoriais e ainda saturados em mais de um fator. Portanto, optou-se pela análise com 2 fatores. As cargas fatoriais de cada um dos fatores apresentaram-se adequadas para posterior análise, conforme mostra a Tabela 3.

Escala Feminina. A Escala Feminina foi analisada com os mesmos critérios da masculina. Apresentou KMO de 0,93 e determinante próximo de zero, indicando a possibilidade de fatoração. Apenas dois fatores apresentaram autovalor acima de 2, mas três fatores com porcentagem de explicação acima de $3 \%$.

Esperando dois fatores, foi analisada a escala desta maneira, e a matriz padrão apresentou cargas fatoriais aceitáveis em dois fatores, conforme Tabela 4. 
Tabela 3

Cargas Fatoriais da junção da SATAQ e da Escala Masculina de Normatização de Padrões de Corpo- EMIC

\begin{tabular}{lcc}
\hline Item $n^{\circ}$ & Item & Cargas \\
& & Fatoriais Comunalidades \\
\hline
\end{tabular}

Fator 1: “Internalização" $(\alpha=0,95)$

13 Eu gostaria de me parecer com os modelos que aparecem de sunga nas revistas

31 Eu gostaria que meu corpo parecesse com o de homens famosos

18 Eu gostaria de parecer tão atlético quanto as pessoas em revistas e na TV

$7 \quad$ Fotografias de homens atléticos me fazem ter vontade de ser atlético

14 Quando eu leio revistas eu me comparo com a aparência dos modelos

26 Os programas de TV e cinema que mostram homens atléticos me fazem querer ser mais atlético

3 Ver homens atléticos me faz ter vontade de ser atlético

1 Os homens que aparecem nos shows de TV e no cinema projetam o tipo de aparência que eu gostaria de ter

5 Eu tendo a comparar o meu corpo com o das pessoas nas revistas e na TV

16 Homens que aparecem na TV e no cinema possuem o tipo de corpo que vejo como meu objetivo

2 Eu acredito que as roupas ficam melhores em homens atléticos

29 Para mim, o ideal seria ter um corpo definido

22 Eu gostaria de parecer tão atlético quanto as atletas profissionais

23 Eu faria cirurgias para melhorar a aparência do meu corpo

28 Eu faria tratamentos estéticos para melhorar a aparência do meu corpo

4 Eu não tenho vontade de ser como as modelos das revistas

11 As pessoas pensam que as roupas vestem melhor quanto mais atlético você está

8 Atratividade é bem importante se você quiser ter sucesso na nossa cultura

9 É importante que pessoas trabalhem duro com seu físico se querem ter sucesso na nossa cultura

$\begin{array}{rr}0,90 & 0,73 \\ 0,89 & 0,71 \\ 0,85 & 0,78 \\ 0,85 & 0,86 \\ 0,83 & 0,73 \\ 0,81 & \\ 0,79 & 0,77 \\ 0,77 & 0,81 \\ 0,75 & 0,57 \\ & 0,66 \\ 0,75 & 0,74 \\ 0,64 & 0,53 \\ 0,53 & 0,77 \\ 0,52 & 0,58 \\ 0,49 & 0,53 \\ 0,46 & 0,65 \\ -0,45 & 0,30 \\ 0,45 & 0,42 \\ 0,44 & 0,40 \\ 0,42 & 0,43\end{array}$

Fator 2: "Intenção de comportamento" $(\alpha=0,90)$

25 Eu faria exercícios para ter menos gordura corporal

27 Atualmente faço exercícios para melhorar minha aparência física

19 Eu faria exercícios para ficar mais definido

Atualmente faço dietas para melhorar minha aparência física

Eu estou sempre tentando melhorar minha aparência corporal

$0,85 \quad 0,63$

$0,80 \quad 0,61$

$0,77 \quad 0,72$

$0,59 \quad 0,55$

$0,56 \quad 0,58$

$\mathrm{Eu}$ faria dietas para ter menos gordura corporal

$0,53 \quad 0,64$

Eu faria dietas para ter um corpo definido

$0,48 \quad 0,78$

Acho importante ter um corpo definido

0,47

0,67

21

Eu compraria produtos para melhorar minha aparência física

0,39

0,54

Tabela 4

Cargas Fatoriais da junção da SATAQ e da Escala Feminina de Normatização de Padrões de Corpo- EFIC

\begin{tabular}{|c|c|c|c|}
\hline Item $n^{\circ}$ & Item & $\begin{array}{c}\text { Cargas } \\
\text { Fatoriais }\end{array}$ & Comunalidades \\
\hline
\end{tabular}

Fator 1: "Internalização" $(\alpha=0,95)$

31 Eu gostaria que meu corpo parecesse com o de mulheres famosas, como atrizes ou modelos

13 Eu gostaria de me parecer como uma modelo que usa biquíni nas revistas

14 Quando eu leio revistas como Nova, Claudia, Vogue eu me comparo com a aparência das modelos

26 Os programas de TV e cinema mostram mulheres magras que me fazem querer ser mais magra

$\begin{array}{ll}0,86 & 0,74 \\ 0,83 & 0,63 \\ 0,82 & 0,68 \\ 0,77 & 0,66\end{array}$


Hirata, E., Pérez-Nebra, A. R. \& Pilati, R. (2012). Desenvolvimento e Validação de Escalas Brasileiras de Percepção e Internalização de Normas Corporais.

16 Mulheres que aparecem na TV e no cinema possuem o tipo de corpo que vejo como meu objetivo

$\begin{array}{rr}0,77 & 0,69 \\ 0,75 & 0,54 \\ 0,73 & 0,74 \\ 0,67 & 0,65 \\ 0,62 & 0,71 \\ 0,55 & 0,54 \\ 0,49 & 0,59 \\ -0,34 & 0,15\end{array}$

Fator 2: "Intenção de comportamento" $(\alpha=0,90)$

Eu faria exercícios para ter menos gordura corporal

$\mathrm{Eu}$ faria exercícios para ficar mais definida

Eu faria dietas para ter um corpo definido

Eu estou sempre tentando melhorar minha aparência corporal

Atualmente faço exercícios para melhorar minha aparência física

Atualmente faço dietas para melhorar minha aparência física

Eu faria dietas para ter menos gordura corporal

0,80

0,74

0,70

0,66

0,63

0,61

Acho importante ter um corpo definido

0,57

0,54

Eu faria tratamentos estéticos para melhorar a aparência do meu corpo

0,48

Eu compraria produtos para melhorar minha aparência física

0,41

0,60

0,56

0,66

0,58

0,40

0,49

0,61

0,53

0,60

O alfa de Internalização foi de 0,95 e de Intenção e Comportamento de 0,90. Este resultado foi similar ao encontrado na escala masculina. Vale notar que os itens são praticamente os mesmos nas duas escalas. A diferença está na escala masculina de internalização, onde itens sobre esforço aparecem com maior freqüência do que no fator de internalização das mulheres.

Relação entre Normas Sociais de Aparência e Variáveis Demográficas. Foram poucas as relações significativas entre as variáveis demográficas e as normas sociais de aparência corporal. Os dados não apontaram diferenças, tanto para homens quanto para mulheres, de a pessoa estar numa relação estável (e.g. estar namorando ou casado e solteiros), da sua etnia, região de residência no país, renda, escolaridade, tipo de trabalho. Incluem-se aqui estudantes e pessoas que não trabalham. Também não houve relações dos fatores com o índice de massa corporal (IMC). Dentre os dados pessoais, o único que apareceu com relação significativa, ainda que com uma relação tímida, foi entre internalização e idade (homens: $r=-0,13 ; p=0,04$; mulheres: $r=-0,13 ; p>0,01)$.

Finalmente, as relações com descrições comportamentais relativas à beleza aparecem significativas com as normas sociais de internalização e intenção e comportamento. Neste caso ler sobre beleza (homensInternalização: $r=0,37 ; p>0,01$; mulheresInternalização: $r=0,26 ; p>0,01$; homensIntenção: $r=0,38 ; p>0,01$; mulheresIntenção: $r=0,31 ; p>0,01)$, comentar sobre beleza com colegas e amigos (homensInternalização: $r=0,40 ; p>0,01$; mulheresInternalização: $r=0,33 ; p>0,01$; homensIntenção: $r=0,39 ; p>0,01$; mulheresIntenção: $r=0,33 ; p>0,01)$ e usar produtos de beleza (homensInternalização: $r=0,32$; $p>0,01$; mulheresInternalização: $r=0,29 ; p>0,01$;
homensIntenção: $r=0,33 ; p>0,01$; mulheresIntenção: $r=0,33 ; p>0,01)$ apresentam relações significativas. Apenas para as mulheres há uma relação significativa dos fatores com a frequência de ida ao salão de beleza (Internalização: $r=0,11 ; p=0,01$; Intenção: $r=0,23$; $p>0,01)$.

\section{Discussão}

O objetivo de validar os instrumentos foi atingido. Entende-se que a escala foi adequada para os dois públicos, já que os alfas foram similares e adequados $(0,95$ para Internalização e 0,90 para Intenção e Comportamento) para a sua utilização no Brasil. O fator de Internalização também é comparável com a escala original do SATAQ, já que nas escalas EMIC e EFIC, mais da metade dos itens compostos neste fator são dos itens originais do SATAQ, o que facilita sua comparação. A adaptação dos itens na escala masculina e sua composição de maneira similar nos fatores revelou que, para este público, os conceitos de atletismo e musculosidade são similares e intercambiáveis. Desta forma conclui-se que a versão híbrida é adequada para uso na pesquisa sobre imagem corporal.

A ausência do fator percepção da norma social pode ser explicada pela própria amostra, que foi de pessoas adultas. A percepção da norma social de aparência pode ser construída ainda muito jovem e na idade adulta ela pode simplesmente não fazer sentido. Outra hipótese explicativa é que ela se construa de maneira diferente do que é descrito pela SATAQ.

As relações entre os fatores e os dados demográficos trazem indicações importantes para a pesquisa na área. Isto porque na maioria dos casos, independente de quem 
seja o indivíduo (seu tipo de trabalho, moradia, relacionamento amoroso, IMC, renda, escolaridade) as normas atuam de maneira similar. A única variável que apresenta relação significativa foi a idade. Neste caso, parece que o aumento da idade diminui a importância subjetiva atribuída às normas de corpo ideal. Assim, quanto mais velha a pessoa, menor será a importância dos padrões de corpo ao self. Esse resultado pode ser interpretado à luz da perspectivas teórica da objetivação. Ao envelhecer, o corpo da mulher vai relativamente perdendo visibilidade, paradoxalmente tornando outras conquistas femininas mais visíveis, como a ênfase na criatividade (Fredrickson \& Roberts, 1997). Ademais, tem-se a literatura de nutrição humana, que relata maior incidência de transtornos alimentares em adolescentes (Philippi \& Alvarenga, 2004).

Os dados de freqüência de comportamento, como ler, comentar e usar produtos de beleza aparecem relacionados tanto com a internalização quanto com a intenção e comportamento. Este dado era esperado, já que tanto a escala quanto os itens tratam de descrições mais próximas do comportamento propriamente dito.

\section{Considerações Finais}

Uma limitação desses estudos foi a amostra de conveniência. Estudos posteriores poderiam incluir amostras aleatórias da população brasileira para testar a estrutura fatorial. Outra possibilidade seria uma análise fatorial confirmatória das escalas. Seria interessante observar se nessas amostras, a mistura de itens de internalização com descrições e intenções de comportamento tornaria a ocorrer.

Apesar das limitações, as medidas mostraram-se válidas e podem servir no entendimento de fatores psicológicos e socioculturais relativos à imagem corporal. O estudo da normatização de corpo, da importância subjetiva dos padrões de corpo ideal e dos comportamentos convergentes a tais padrões pode ser útil na sociedade brasileira atual.

Uma ação de pesquisa necessária diz respeito ao desenvolvimento do fator de percepção de normas sociais. Outra questão relevante é conhecer outros fatores individuais que estejam relacionados à internalização e a intenção e comportamento, já que são estes fatores que sugerem intervenção nos diversos ambientes clínicos, sociais e gerenciais. Finalmente, um estudo mais detalhado sobre a relação entre a idade e a norma social se faz necessário para entender o fenômeno. Dado o achado da relação entre idade e internalização, seria identificar as variáveis que podem estar por trás da redução da importância desses padrões em idades mais avançadas. Tal estudo poderia auxiliar no desenvolvimento de estratégias de enfrentamento para indivíduos com alto grau de internalização de padrões.

De forma geral considera-se que as escalas aqui apresentadas indicam validade e podem ser utilizadas em pes- quisas para o estudo de questões relativas à imagem corporal.

\section{Referências}

Aarts, H., Dijkesterhuis, A., \& Custers, R. (2003). Automatic normative behavior in environments: The moderating role of conformity in activating situational norms. Social Cognition, 21, 447-464.

Baumeister, R. F. (1998). The self. In D. T. Gilbert, S. T. Fiske, \& G. Lindzey (Eds.), The Handbook of Social Psychology ( $4^{\text {th }}$ ed., pp. 680-740). New York: McGraw-Hill.

Bessenoff, G. R., \& Snow, D. (2006). Absorbing society's influence: Body image self-discrepancy and internalized shame. Sex Roles, 54, 727-773.

Cafri, G., Yamamiya, Y., Brannick, M., \& Thompson, J. K. (2005). The influence of sociocultural factors on body image: A meta-analysis. Clinical Psychology: Science and Practice, 12, 421-433.

Cash, T. F. (2000). User's manual for the Multidimensional Body-Self Relations Questionaire. Norfolk, VA: Old Dominion University.

Cialdini, R. B., \& Goldstein, N. (2004). Social influence: Compliance and conformity. Annual Review of Psychology, 55, 591-621.

Cialdini, R. B., \& Trost, M. R. (1998). Social influence: Social norms, conformity, and compliance. In D. T. Gilbert, S. T. Fiske, \& G. Lindzey (Eds.), The Handbook of Social Psychology (4 ${ }^{\text {th }}$ ed., Vol. 2, pp. 151-192). New York: McGraw-Hill.

Deutsch, M., \& Gerard, H. B. (1955). A study of normative and informational social influences upon individual judgment. The Journal of Abnormal and Social Psychology, 51(3), 629-636.

Dittmar, H. (2008). Consumer culture, identity and well-being: The search for the 'good life' and the 'body perfect'. Canterbury, UK: Psychology Press.

Dittmar, H., \& Howard, S. (2004). Thin-ideal internalization and social comparison tendency as moderators of media model's impact on women's body-focused anxiety. Journal of Social and Clinical Psychology, 23, 768-791.

Durkin, S. J., \& Paxton, S. J. (2002). Predictors of vulnerability to reduced body image satisfaction and psychological wellbeing in response to exposure to idealized female media images in adolescent girls. Journal of Psychosomatic Research, 53, 995-1005.

Durkin, S. J., Paxton, S. J., \& Sorbello, M. (2007). An integrative model of the impact of exposure to idealized female images on adolescent girls' body satisfaction. Journal of Applied Social Psychology, 37, 1092-1117.

Enzmann, D. (1997). RanEigen: A program to determine the parallel analysis criterion for the number of principal components. Applied Psychological Measurement, 21, 232.

Etcoff, N. (1999). Survival of the prettiest. The science of beauty. Boston: Anchor Books.

Ford, T. E., Stevenson, P. R., Wienir, P. L., \& Wait, R. F. (2002). The role of internalization of gender norms in regulating Selfevaluations in response to anticipated delinquency. Social Psychology Quarterly, 65, 202-212.

Fredrickson, B. L., \& Roberts, T. A. (1997). Objectification theory: Toward understanding women's lived experiences and mental health risks. Psychology of Women Quarterly, 21, 173-206. 
Forbes, G. B., Adam-Curtis, L., Jobe, R. L., White, K. B., Reveak, J., Zivcic-Beciveric, I., et al. (2005). Body dissatisfaction in college women and their mothers: Cohort effects, developmental effects, and the influences of body size, sexism, and the thin body ideal. Sex Roles, 53, 281298.

Giavoni, A., \& Tamayo, A. (2005). Inventário feminino dos esquemas de gênero do autoconceito (IFEGA). Estudos de Psicologia (Natal), 10, 25-34.

Groesh, L. M., Levine, M. P. \& Murnen, S. K. (2002). The effect of experimental presentation of thin media images on body satisfaction: A meta-analytic review. International Journal of Eating Disorders, 31, 1-16.

Grogan, S. (2008). Body image: Understanding body dissatisfaction in men, women, and children. East Sussex, UK: Routledge.

Harrison, K., Taylor, L. D., \& Marsk, A. L. (2006). Women's and men's eating behavior following exposure to ideal-body images and text. Communication Research, 33, 507-529.

Harvey, J. A., \& Robinson, J. D. (2003). Eating disorders in men: Current considerations. Journal of Clinical Psychology in Medical Settings, 10, 297-306.

Heinberg, L. J., Thompson, J. K., \& Stormer, S. (1995). Development and validation of the Sociocultural Attitudes Towards Apperance Questionnaire. International Journal of Eating Disorders, 17, 81-89.

Hirata, E. (2009). Influências de padrões de corpo e da comparação social na imagem corporal. (Dissertação de Mestrado não-publicada). Universidade de Brasília, DF.

Hirata, E., \& Pilati, R. (2010). Desenvolvimento e validação preliminar da Escala Situacional de Satisfação CorporalESSC. Psico-USF, 15, 1-11.

Jackson, L. (2002). Physical attractiveness: A sociocultural perspective. In T. F. Cash \& T. Pruzinsky (Eds.), Body image: $A$ handbook of theory, research and clinical practice (pp. 13-21). New York: The Guillford Press.

Junta Internacional de Fiscalização de Entorpecentes. (2007). Relatório Anual da Junta Internacional de Fiscalização de Entorpecentes - JIFE. Retrieved February 4, 2009 from http:/ /www.unodc.org/pdf/brazil/JIFE/PricipaisPontosJIFE.pdf

Labre, M. P. (2002). Adolescent boys and the muscular male body ideal. Journal of Adolescent Health, 30, 233-242.

McKinley, N. M. (2002). Feminist perspectives and objectified body consciousness. In T. F. Cash \& T. Pruzinsky (Eds.), Body image: A handbook of theory, research and clinical practice (pp. 55-62). New York: The Guillford Press.

Park, S-Y. (2005). The influence of presumed media on women's desire to be thin. Communication Research, 32, 594-614.

Pasquali, L. (2003). Psicometria: Teoria dos testes na Psicologia e na Educação. Petrópolis, RJ: Vozes.

Philippi, S. T., \& Alvarenga, M. (2004). Transtornos alimentares: Uma visão nutricional. São Paulo, SP: Manole.

Ryan, R. M., \& Connell, J. P. (1989). Perceived locus of causality and internalization: Examining reasons for acting in two domains. Journal of Personality and Social Psychology, 57, 749-761.

Sociedade Brasileira de Cirurgia Plástica. (2004). Boletim Press Release. Retrieved January 29, 2009, from http:// www.cirurgiaplastica.org.br/publico/press01.cfm

Strahan, E. J. Spencer, S. J., \& Zanna, M. P. (2007). Don't take another bite: How sociocultural norms for appearance affect women's eating behavior. Body Image, 4, 331-342.
Snijders, T. A. B. (1992). Estimations on the basis of snowball samples: How to weight? Bulletin de Méthodologie Sociologique, 36, 59-70.

Tabachnick, B., \& Fidell, L. (2007). Using multivariate statistics $\left(5^{\text {th }}\right.$ ed.). Nova York: HarperCollins.

Tamayo, A. (1981). EFA: Escala fatorial de autoconceito. Arquivo Brasileiro de Psicologia, 33, 87-102.

Thompson, J. K., \& Stice, E. (2001). Thin-ideal internalization: Mounting evidence for a new risk factor for body-image disturbance and eating pathology. Current Directions in Psychological Science, 10, 181-183.

Thompson, J. K., Van den Berg, P., Roehrig, M., Guarda, A. S., \& Heinberg, L. J. (2004). The Sociocultural Attitudes Towards Appearance Scale-3 (SATAQ-3): Development and validation. International Journal of Eating Disorders, 53, 293-304

Tiggemann, M., \& McGill, B. (2004). The role of social comparison in the effect of magazine advertisements on women's mood and body dissatisfaction. Journal of Social and Clinical Psychology, 23, 23-44.

Tovée, M. J., Hancock, P. J. B., Mahmoodi1, S., Singleton, B. R. R., \& Cornelissen, P. L. (2002). Human female attractiveness: Waveform analysis of body shape. Proceedings of the Royal Society of London B, 269, 2205-2213. 